\title{
Variation in the ovine WFIKKN2 gene
}

\author{
Jiqing Wang ${ }^{\mathrm{a}}$, Huitong Zhou ${ }^{\mathrm{b}}$, Qian Fang ${ }^{\mathrm{b}}$, Yuzhu Luo ${ }^{\mathrm{a}, *}$, Jon G.H. Hickford ${ }^{\mathrm{b}, *}$ \\ a Gansu Key Laboratory of Herbivorous Animal Biotechnology, Faculty of Animal Science and Technology, Gansu Agricultural University, Lanzhou 730070, China \\ b Gene-Marker Laboratory, Faculty of Agriculture and Life Sciences, PO Box 84, Lincoln University, Lincoln 7647, New Zealand
}

\section{A R T I C L E I N F O}

\section{Article history:}

Received 19 December 2013

Received in revised form 26 February 2014

Accepted 31 March 2014

Available online 3 April 2014

\section{Keywords:}

WFIKKN2

Variation

PCR-SSCP

Sheep

\begin{abstract}
A B S T R A C T
WFIKKN2 may play a role in the regulation of muscle growth and development through its interaction with growth and differentiation factor 8 (GDF8) and growth and differentiation factor 11 (GDF11), but to date research into the function of the protein has been focused on mice, even though the WFIKKN2 gene (WFIKKN2) was first identified in humans in 2001.

In this study two regions (intron 1 and the 3' UTR) of ovine WFIKKN2 were investigated, using Polymerase Chain Reaction-Single Stranded Conformational Polymorphism (PCR-SSCP). Two different PCR-SSCP patterns, representing two unique DNA sequences (designated $a$ and $b$ ) were detected in a 399-bp amplicon derived from the 3' UTR, with sequence analysis revealing one single nucleotide polymorphism (SNP). In a 421-bp amplicon from intron 1, five different PCR-SSCP patterns (designated $A-E$ ) were observed and twelve SNPs were detected. Either one or two different sequences were detected in individual sheep and all the sequences identified shared homology with the WFIKKN2 sequences from cattle and other animal species, suggesting that these sequences represent variants of the ovine WFIKKN2 gene. In intron 1 of 487 sheep from eight breeds, variants $B$ and $C$ were the most common, followed by $A, D$ and $E$. These results indicate that ovine WFIKKN2 is polymorphic and suggest that further analysis is required to see if variation in the gene is associated with variation in growth and muscle traits in sheep.
\end{abstract}

(c) 2014 Elsevier B.V. All rights reserved.

\section{Introduction}

WFIKKN2 (also called growth and differentiation factor associated serum protein-1, GASP1) is a large extracellular multi-domain protein that contains a whey acidic protein (WAP)-domain, a follistatin-domain, an immunoglobulin-domain, two Kunitz-type protease inhibitordomains and a NTR-domain (Trexler et al., 2001). WFIKKN2 was first identified in humans in 2001 (Trexler et al., 2001), but many aspects of its biology in human and livestock species are not understood.

In vitro experiments reveal that WFIKKN2 is a potent inhibitor of two TGF beta family members that are implicated in musculoskeletal development: GDF8 (growth and differentiation factor 8, also known as myostatin) and GDF11 (growth and differentiation factor 11, also known as bone morphogenetic protein 11) (Hill et al., 2003; Kondas et al., 2008). Specifically, $3 \mathrm{nM}$ and $50 \mathrm{nM}$ WFIKKN2 cause 50\% and 90\% inhibitions of GDF8 and GDF11 activities respectively, using a SPR (surface plasmon resonance) assay in a solution-competition format (Szlama et al., 2010).

Abbreviations: PCR, polymerase chain reaction; SSCP, single-stranded conformational polymorphism; GASP1, growth and differentiation factor associated serum protein-1; GDF, growth and differentiation factor; BMP, bone morphogenetic protein; SPR, surface plasmon resonance; SIL, sheep improvement limited.

* Corresponding authors.

E-mail addresses: luoyz@gsau.edu.cn (Y. Luo), Jon.hickford@lincoln.ac.nz (J.G.H. Hickford).
GDF8 acts primarily as a negative regulator of muscle development in mammals. Deletions or mutations in the GDF8 gene can cause an increase in skeletal muscle mass as a result of both muscle hypertrophy and hyperplasia in a number of animal species including sheep (Clop et al., 2006), cattle (Gill et al., 2009; Kambadur et al., 1997) and dog (Mosher et al., 2007). GDF11 also appears to play a crucial role in skeletal development, as knock-out mice display skeletal defects resulting from abnormal anterior-posterior patterning (McPherron et al., 1999).

The expression of WFIKKN2 in different tissues has been described in humans and mice. In the developing human foetus, WFIKKN2 is expressed primarily in skeletal muscle (Trexler et al., 2002), and high levels of expression of WFIKKN2 have also been reported in the skeletal muscle of mice (Hill et al., 2003; Lee and Lee, 2013; Marcelo et al., 2009). Haidet et al. (2008) found that in vivo viral delivery of a WFIKKN2 expression cassette into adult mice hind-limb and fore-limb muscle significantly increased overall body mass and the mass of the Tibialis anterior, Gastrocnemius, Quadriceps and Triceps muscles.

Monestier et al. (2012) observed that the over-expression of WFIKKN2 caused obvious increases in muscle mass resulting from hypertrophy rather than hyperplasia in a gain-of-function transgenic mouse model, and Lee and Lee (2013) further confirmed the effect of WFIKKN2 on muscle weight, describing how mice genetically engineered to lack WFIKKN2 had a shift in muscle fibre type from fast glycolytic typellb to fast oxidative typeIla, as well as having impaired muscle regeneration ability. Taken 
together, this literature suggests that WFIKKN2 may be important in the regulation of muscle mass.

Variation in the WFIKKN2 gene has been recorded for humans, cattle, pigs and chickens based on analysis of DNA sequences in GenBank and Ensembl, although the sequence variations observed have not been confirmed in published reports. This suggests that ovine WFIKKN2 may be polymorphic and thus the objective of this study was to identify if variation occurs in ovine WFIKKN2, and in anticipation of a much larger study investigating whether variation in WFIKKN2 if found, has an effect on ovine growth and muscle traits.

\section{Materials and methods}

\subsection{Sheep investigated and DNA collection}

A total of one hundred not closely related sheep, selected from a variety of common breeds in New Zealand (NZ) including Corriedale $(n=12)$, Dorper $(n=16)$, Dorset Down $(n=16)$, Poll Dorset $(n=$ $18)$, Perendale $(n=16)$ and Merino $(n=22)$, were used initially to identify variation in portions of intron 1 and the 3' UTR of ovine WFIKKN2.

Having identified variation in intron 1 of WFIKKN2, 487 sheep from eight breeds were used to calculate variant frequency. These comprised 422 sheep from 29 farms in New Zealand, including Corriedale $(n=48)$, Dorper $(n=60)$, Dorset Down $(n=37)$, Poll Dorset $(n=33)$, Perendale $(n=21)$, Merino $(n=62)$ and NZ Romney $(n=161)$, plus 65 Tibetan sheep samples from three farms in the Gansu Province of China. All these sheep were not closely related with the exception of NZ Romneys that were the progeny of five not closely related NZ Romney rams. Genomic DNA was purified from blood collected on FTA cards (Whatman BioScience, Middlesex, UK) according to the method described by Zhou et al. (2006).

\subsection{PCR amplification and SSCP analysis}

Two sets of PCR primers were designed for amplification of either a portion of intron 1 or the 3' UTR of ovine WFIKKN2, based on the bovine gene (ENSBTAG00000000731). These primers are described in Table 1. The primers were synthesised by Integrated DNA Technologies (Coralville, IA, USA).

Amplifications were performed in a $20 \mu \mathrm{L}$ reaction consisting of the DNA on one $1.2 \mathrm{~mm}$ punch of FTA card, $2.5 \mu \mathrm{L}$ of $10 \times$ PCR buffer (Qiagen, Hilden, German), $0.25 \mu \mathrm{M}$ of each primer, $150 \mu \mathrm{M}$ dNTPs (Bioline, London, UK), $2.5 \mathrm{mM} \mathrm{Mg}^{2+}$ (Qiagen), $0.5 \mathrm{U}$ Taq DNA polymerase (Qiagen) and $\mathrm{dd}_{2} \mathrm{O}$ to make up volume. The thermal profile for the two regions amplified consisted of $2 \mathrm{~min}$ at $94{ }^{\circ} \mathrm{C}$, followed by 35 cycles of $30 \mathrm{~s}$ at $94^{\circ} \mathrm{C}, 30 \mathrm{~s}$ at annealing temperature shown in Table 1 and $30 \mathrm{~s}$ at $72{ }^{\circ} \mathrm{C}$, with a final extension of $5 \mathrm{~min}$ at $72{ }^{\circ} \mathrm{C}$. Amplification was carried out in Bio-Rad S1000 thermal cyclers (Bio-Rad, Hercules, CA, USA).

The PCR amplicons were visualized by electrophoresis in $1 \%$ agarose (Quantum Scientific, Queensland, Australia) gels, using $1 \times$ TBE buffer [89 mM Tris (Thermo Fisher, MA, USA), 89 mM boric acid (Thermo Fisher), $2 \mathrm{mM} \mathrm{Na}_{2}$ EDTA (Thermo Fisher)] containing $200 \mathrm{ng} / \mathrm{mL}$ of ethidium bromide (Bio-Rad). A $2 \mu \mathrm{L}$ aliquot of PCR product was added to $2 \mu \mathrm{L}$ of loading dye [0.2\% bromophenol blue (Bio-Rad), $0.2 \%$ xylene-cyanol (Thermo Fisher), 40\% (w/v) sucrose (Quantum Scientific)] and the gel was run at a constant $10 \mathrm{~V} / \mathrm{cm}$ for $10 \mathrm{~min}$, prior to visualization by UV transillumination at $254 \mathrm{~nm}$.

For SSCP analysis, a $0.7 \mu \mathrm{L}$ aliquot of each amplicon was mixed with $7 \mu \mathrm{L}$ of loading dye [98\% formamide (Fisher Scientific, Loughborough, UK), 10 mM EDTA (Thermo Fisher), 0.025\% bromophenol blue (BioRad), $0.025 \%$ xylene-cyanol (Thermo Fisher)] and after denaturation at $95{ }^{\circ} \mathrm{C}$ for $5 \mathrm{~min}$, samples were rapidly cooled on ice and then loaded on $16 \times 18 \mathrm{~cm}, 14 \%$ acrylamide:bisacrylamide (37.5:1) (Bio-Rad) gels. Electrophoresis was performed using Protean II xi cells (Bio-Rad) for $19 \mathrm{~h}$ in $0.5 \times$ TBE at conditions described in Table 1 . Gels were silverstained according to the method of Byun et al. (2009).

\subsection{Sequencing of amplicons}

Amplicons that were identified as homozygous by PCR-SSCP were directly sequenced at the Lincoln University DNA Sequencing Facility. Those sequences only found in a heterozygous form were sequenced using a rapid sequencing approach that has been described previously (Gong et al., 2011).

\subsection{Sequence analyses}

Hardy-Weinberg equilibrium was calculated using POPGENE version 3.2 (Molecular Biology and Biotechnology Centre, University of Alberta, Canada). Sequence alignment and comparison were carried out using DNAMAN version 5.2.10 (Lynnon BioSoft, Vaudreuil, Canada). The BLAST algorithm was used to search the NCBI GenBank (http:// www.ncbi.nlm.nih.goc/) databases for homologous sequences.

A neighbour-joining phylogenetic tree was constructed on the basis of genetic distances in intron 1 of ovine WFIKKN2, estimated by the Kimura two-parameter method, using MEGA version 4.0 (Tamura et al., 2007). The reliability of the trees was estimated by bootstrap confidence values, and 1000 bootstrap replications were used.

Sequences for phylogenetic analysis included horse (ENSECAG00000015064), pig (ENSSSCG00000017557), dog (ENSCAFG00000017224) and bovine (ENSBTAG00000000731), together with the ovine WFIKKN2 variant sequences identified in this study. The sequences were trimmed to a length corresponding to the PCR amplicon before generating the neighbour-joining tree.

\section{Results}

\subsection{Identification of variants of ovine WFIKKN2}

An amplicon of 399-bp from the 3' UTR were obtained from all 100 samples from the initial six breeds. Two unique PCR-SSCP patterns (Fig. 1a) representing two unique DNA sequences (designated $a$ and $b$ ) were detected, but only in the Poll Dorset samples. A BLAST search of GenBank revealed that the two variant sequences showed high similarity to the WFIKKN2 sequences reported from cattle, Tibetan antelope and horse. Variant $a$ was identical to the ovine genome assembly v3.1, while $b$ had one nucleotide difference to it. These ovine sequences were deposited into GenBank with accession numbers KF824754-KF824755. One single nucleotide polymorphism (SNP) (c. ${ }^{*} 600 \mathrm{G}>\mathrm{T}$ ) was observed. There were $17 a a(94.44 \%)$ and $1 a b(5.56 \%)$ in the original 18 Poll Dorsets, with a frequency of $97.22 \%$ and $2.78 \%$ for $a$ and $b$ respectively.

Table 1

The primer sequences and SSCP conditions for amplicons of ovine WFIKKN2.

\begin{tabular}{|c|c|c|c|c|c|}
\hline & Primer binding region ${ }^{\mathrm{a}}$ & Primer sequence $\left(5^{\prime}-3^{\prime}\right)$ & Amplicon size & PCR annealing temperature & SSCP condition \\
\hline \multirow[t]{2}{*}{ Intron 1} & g.1000_1018 & GAGACGGACCAGGTGAGTG & 421 bp & $58^{\circ} \mathrm{C}$ & $220 \mathrm{~V}, 14 \%, 25^{\circ} \mathrm{C}$ \\
\hline & g.1391_1410 & AGAGGCTGGATGAAGCATCG & & & \\
\hline \multirow[t]{2}{*}{ 3' UTR } & g.5522_5542 & AGCACTATGAACAGAGACCAC & 399 bp & $58^{\circ} \mathrm{C}$ & $220 \mathrm{~V}, 14 \%, 33^{\circ} \mathrm{C}$ \\
\hline & g.5901_5920 & TTATCTGCTTCTGGGAGAGC & & & \\
\hline
\end{tabular}

\footnotetext{
a The primer binding position is given relative to the bovine WFIKKN2 DNA sequences (ENSBTAG00000000731).
} 
(a)
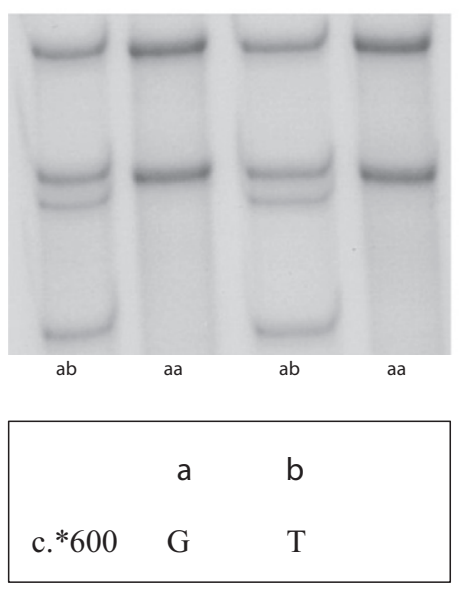

(b)

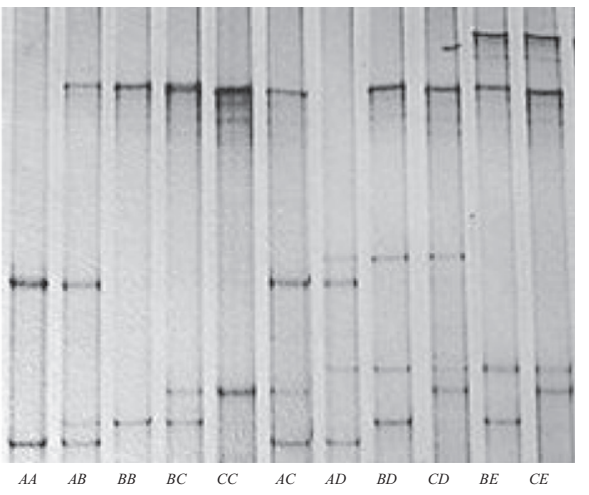

\begin{tabular}{|llllll|}
\hline & $A$ & $B$ & $C$ & $D$ & $E$ \\
c. $204+79$ & A & G & G & A & A \\
c. $204+116$ & C & A & C & C & C \\
c. $204+155$ & G & G & A & G & G \\
c. $204+169$ & A & G & A & A & A \\
c. $204+178$ & G & G & G & A & G \\
c. $204+229$ & A & G & G & A & G \\
c. $204+230$ & G & A & G & G & G \\
c. $204+261$ & A & A & A & A & G \\
c. $204+289$ & A & G & G & A & G \\
c. $204+318$ & T & C & C & T & C \\
c. $204+323$ & C & T & C & C & C \\
c. $204+365$ & A & C & A & A & A \\
\hline
\end{tabular}

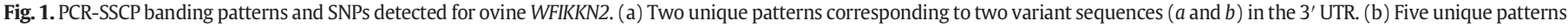

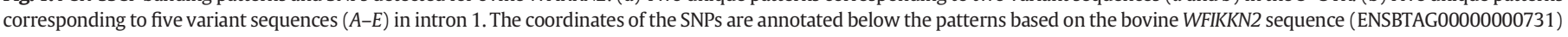
and the numbering of SNPs follows the guidelines at http://www.hgvs.org/mutnomen/.

Overall in the 100 sheep, $a a$ (99\%) and $a b$ (1\%) were observed, with frequencies of $99.5 \%$ and $0.5 \%$ for $a$ and $b$ respectively.

In a 421-bp amplicon from intron 1 of ovine WFIKKN2, five unique PCR-SSCP patterns (Fig. 1b) representing five DNA sequences were identified in the 100 sheep. These sequences were named $A-E$ and deposited into the GenBank with the accession numbers KF824756KF824760. None of these sequences was identical to the ovine genome assembly v 3.1, but all shared over $98 \%$ homology to it. There were a total of twelve SNPs identified among the five variants (c.204+ 79G >A, c.204 + 116C >A, c.204+155G>A, c.204+169A >G, c. $204+178 \mathrm{G}>$ A, c.204 + 229G $>$ A, c.204 + 230A $>$ G, c. $204+261 \mathrm{~A}>\mathrm{G}$, c. $204+289 G>A$, c. $204+318 C>$ T, c. $204+323 \mathrm{C}>$ T and c.204 + $365 \mathrm{~A}>\mathrm{C}$, see Fig. 1b). Phylogenetic analysis of these intron 1 sequences and sequences from cattle, pig, horse and dog, revealed that the five variant sequences shared high similarity with the WFIKKN2 sequences from cattle and other animal species. The variant sequences of ovine WFIKKN2 could be clustered into two clades, with $B$ belonging to one clade and the remaining variants in the other clade (Fig. 2).

\subsection{Variant frequencies and Hardy-Weinberg equilibrium for intron 1 in the 487 sheep from eight breeds}

The frequencies of the variants of intron 1 of ovine WFIKKN2 in the 487 sheep from eight breeds are summarised in Table 2. Overall, $B$ and $C$ were the most common variants in the study, with an overall frequency of $30.60 \%$ and $43.33 \%$, respectively, followed by $A$ with an overall frequency of $21.25 \%$. $D$ and $E$ were the least common variants with an overall frequency of $2.46 \%$ and $2.46 \%$ respectively. The genotype distributions for intron 1 displayed deviation from Hardy-Weinberg equilibrium in the NZ Romney sheep $(P<0.01)$, but were in equilibrium in all of the remaining breeds $(P>0.05)$ (Table 2$)$.

\section{Discussion}

This is the first study to report sequence variation in ovine WFIKKN2. As no complete ovine WFIKKN2 sequence was yet available when the study commenced, we had to design primers to amplify two regions (intron 1 and the $3^{\prime}$ UTR) of the gene based on the published bovine sequences. In both regions investigated, either one or a combination of two different SSCP patterns was observed for each sheep, which was consistent with there being homozygous and heterozygous genotypes,

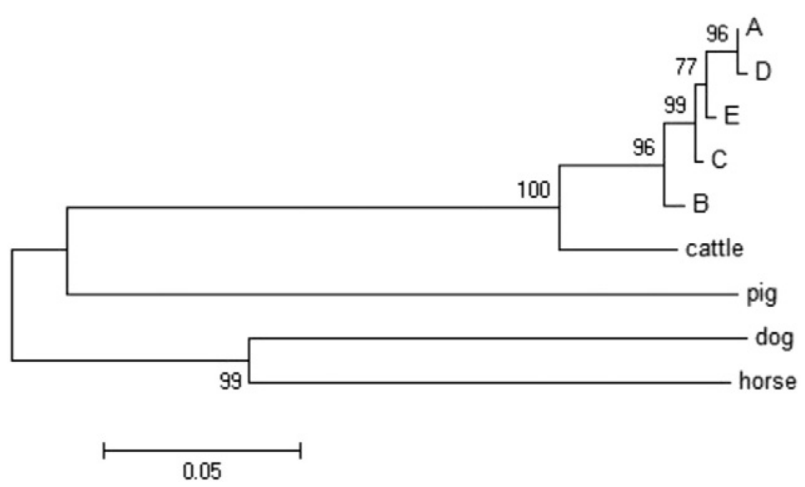

Fig. 2. Neighbour-joining tree for intron 1 sequences of WFIKKN2 from sheep, cattle, pig, dog and horse. The numbers at the forks indicate the bootstrap confidence values and branch lengths are proportional to genetic distance. 
Table 2

Variant frequencies for intron 1 of ovine WFIKKN2 in various breeds.

\begin{tabular}{|c|c|c|c|c|c|c|c|}
\hline \multirow[t]{2}{*}{ Breeds } & \multirow[t]{2}{*}{$n$} & \multicolumn{5}{|c|}{ Variant frequency (\%) } & \multirow[t]{2}{*}{$\mathrm{HWE}^{\mathrm{a}}$} \\
\hline & & $A$ & $B$ & $C$ & $D$ & $E$ & \\
\hline NZ Romney & 161 & 31.37 & 54.97 & 11.18 & 0.93 & 1.55 & 0.00 \\
\hline Merino & 62 & 4.84 & 10.48 & 70.97 & 2.42 & 11.29 & 0.44 \\
\hline Poll Dorset & 33 & 9.09 & 9.09 & 80.30 & 1.52 & 0.00 & 0.94 \\
\hline Corriedale & 48 & 28.12 & 13.54 & 51.04 & 6.25 & 1.04 & 0.77 \\
\hline Perendale & 21 & 19.05 & 35.71 & 40.48 & 0.00 & 4.76 & 0.61 \\
\hline Dorper & 60 & 35.83 & 13.33 & 48.33 & 1.67 & 0.83 & 0.78 \\
\hline Dorset Down & 37 & 20.27 & 8.11 & 70.27 & 1.35 & 0.00 & 0.20 \\
\hline Chinese Tibetan sheep & 65 & 0.00 & 40.00 & 53.08 & 6.15 & 0.77 & 0.85 \\
\hline Overall & 487 & 21.25 & 30.60 & 43.33 & 2.46 & 2.46 & \\
\hline
\end{tabular}

${ }^{\text {a }} P$ value for testing Hardy-Weinberg equilibrium (HWE).

respectively. All the sequences identified shared high homology to the WFIKKN2 sequences from cattle and other animal species. These results suggest that these sequences represent variants of the ovine WFIKKN2 gene, and that sheep have a single WFIKKN2 gene, rather than the two WFIKKN2-related genes reported in fish genomes (Kondas et al., 2008).

Five and two variants were identified in intron 1 and the $3^{\prime}$ UTR of WFIKKN2 respectively, suggesting that the gene is polymorphic in sheep. Given that only a portion of intron 1 or the 3' UTR was amplified, more variants probably exist when an extended gene region is analysed, and further variation should be in linkage with that reported here. It should also be noted that the majority of the sheep investigated were sourced from within New Zealand, with the exception of Chinese Tibetan sheep. It could therefore be expected that different variants may be found when additional sheep from more breeds around the world are investigated.

It is noteworthy that there were twelve SNPs in the 421-bp amplified region of intron 1 . When these DNA sequences were aligned, it was revealed that five SNPs (c.204 + 116C>A, c.204 + 169A>G, c. $204+230 A>G$, c. $204+323 C>$ T and c. $204+365 A>C$ ) were in linkage and were unique to variant $B$. In contrast, c. $204+155 \mathrm{G}>\mathrm{A}$, c. $204+178 \mathrm{G}>\mathrm{A}$ and c. $204+261 \mathrm{~A}>\mathrm{G}$ were unique to $C, D$ and $E$, respectively. There was only a single nucleotide difference between $A$ and $D$ (c.204 $+178 \mathrm{G}>\mathrm{A})$. When compared to the bovine WFIKKN2 DNA sequence (ENSBTAG00000000731), there was a deletion (c.204 + 110delG) and an insertion (c.204 + 324_c.204 + 325insCTGCATGGAAG) in the ovine variants of intron 1, suggesting that these may be sequence differences between cattle and sheep.

Although the variation identified here is located in intron 1, it may be linked to other variations in critically important regions of the gene that regulate expression. The first intron of other genes has been postulated to modify expression levels and affect transcription efficiency by means of possessing important regulatory elements, such as enhancers, silencers or other elements that modulate the function of the main upstream promoter (Chorev and Carmel, 2012; Gaunitz et al., 2004; Greenwood and Kelsoe, 2003). Furthermore, the association between variation in intron 1 with variation in livestock traits has been reported previously (Hickford et al., 2010; Zhang et al., 2012).

In the two regions studied, polymorphism in the 3' UTR was low with $a$ and $b$ having an overall frequency of $99.5 \%$ and $0.5 \%$ respectively. Given this low variation, only the initial 100 samples were analysed for variation in the $3^{\prime}$ UTR. Based on the variants identified in both intron 1 and the 3' UTR in the 100 samples typed first, seven haplotypes of ovine WFIKKN2 could be deduced. These were $A a, B a, C a, D a, E a$, and $A b$ and $C b$.

It is notable that of the eight sheep breeds investigated for intron 1 variation, only NZ Romney sheep failed to meet the requirement of being in Hardy-Weinberg equilibrium $(P<0.01)$. This may be a consequence of strong artificial selection, as the 161 Romneys studied were progeny of sires, which are ranked in the top 20\% of NZ Romney rams in New Zealand based on the Sheep Improvement Limited (SIL - a Division of Meat and Wool NZ, Wellington, NZ)-Dual Purpose Overall (DPO) index. This index is based on measuring genetic merit for a variety of traits of value and has a widespread use in the NZ sheep breeding industry.

The $C$ variant was the most common in Merino, Poll Dorset and Corriedale sheep. While the Corriedale sheep is known to have been bred in North Canterbury, New Zealand from Merino sheep, the origin of the Poll Dorset is less certain. It is speculated that Merino sheep and horned Dorset sheep share an ancestral link, based on the observation that both have an extended breeding season. In contrast, while the Perendale sheep is known to be a Cheviot-Romney cross, the variant frequencies in the two breeds appear to be quite different, with $A$ and $B$ as the most common variants in the NZ Romney sheep, while in Perendale sheep the most common variants were $B$ and $C$. Care is needed in this interpretation as the sheep typed were not chosen so as to necessarily be representative of the breeds as a whole.

Some variants were not found in some of the breeds. In the 3' UTR region, the $b$ variant was only found in Poll Dorset sheep. Care is needed in pronouncing this variant to be breed-specific as once again the sheep investigated were not necessarily representative of all worldwide sheep breeds. In intron 1, compared with a relatively high frequency of $A$ in the NZ breeds, there was an absence of $A$ in the Chinese Tibetan sheep. This too may be breed-specific, as may be the lack of $D$ and $E$ in Perendales, Poll Dorsets and Dorset Down sheep, although only small numbers of animals from each breed were studied.

Although no obvious patterns in variant frequency were observed with respect to the recognised wool breed (Merino), meat breed (Poll Dorset, Dorper and Dorset Down) or dual-purpose breed (Romney, Corriedale, Perendale and Chinese Tibetan sheep), the distribution of individual variants seems to be related to generalised production traits in some breeds. For example, $E$ was the second most common variant (11.29\%) in Merino sheep, while it was rare in the other breeds $(0.00-$ 4.76\%). It may therefore be associated with unique traits (such as wool traits) in Merino sheep. WFIKKN2 can bind bone morphogenetic protein 2 (BMP2) and bone morphogenetic protein 4 (BMP4) with relatively high affinity (Szlama et al., 2010), and the genes for these two proteins are expressed in hair shaft precursor cells (Kulessa et al., 2000), while ectopic expression of BMP2 or BMP4 suppresses the formation of feather buds in chick embryos (Jung et al., 1998; Noramly and Morgan, 1998). Additionally, because WFIKKN2 possesses a follistatin-domain, it may have an overlapping function with follistatin, which has been shown to be an important regulator of cell proliferation, differentiation and apoptosis in hair follicle initiation, hair cycling, normal skin homeostasis and wound healing (McDowall et al., 2008). This speculation would need to be investigated by looking for associations between WFIKKN2 variation and variation in wool traits.

Given that WFIKKN2 interacts with GDF8 and GDF11, and because these factors play significant roles in the regulation of muscle development, it might be inferred that WFIKKN2 has an effect on growth or muscle traits in sheep. In this respect it is notable that $A$ exists at a higher frequency (4.84-35.83\%) in the NZ sheep, but is absent in the Chinese Tibetan sheep. Chinese Tibetan sheep tend to be slower growing than NZ sheep because of feed availability in the China-Tibetan plateau region, but this may also reflect genetic differences including differences in WFIKKN2. This too will require much additional investigation with more sheep and breeds. The $A$ variant was also not present at high frequency in Merino sheep, a breed that is slow growing in the NZ context (Muir et al., 1992). Together, this suggests that WFIKKN2 is worthy of further study in the context of variation in growth and muscle traits.

\section{Conflict of interest}

The authors declare that there is no conflict of interest.

\section{Acknowledgements}

We acknowledge the financial support of the Projects of International Cooperation and Exchanges of China (2011DFG33310) and the 
Lincoln University Gene-Marker Laboratory. The authors would like to thank Seung OK Byun and Hua Gong for the technical assistance during experiments.

\section{Appendix A. Supplementary data}

Supplementary data to this article can be found online at http://dx. doi.org/10.1016/j.gene.2014.03.062.

\section{References}

Byun, S.O., Fang, Q. Zhou, H., Hickford, J.G.H., 2009. An effective method for silver-staining DNA in large numbers of polyacrylamide gels. Analytical Biochemistry 385, 174-175.

Chorev, M., Carmel, L., 2012. The function of introns. Frontiers in Genetics 3, 1-15.

Clop, A., et al., 2006. A mutation creating a potential illegitimate microRNA target site in the myostatin gene affects muscularity in sheep. Nature Genetics 38, 813-818.

Gaunitz, F., Heise, K., Gebhardt, R., 2004. A silencer element in the first intron of the glutamine synthetase gene represses induction by glucocorticoids. Molecular Endocrinology 18, 63-69.

Gill, J.L., Bishop, S.C., McCorquodale, C., Williams, J.L., Wiener, P., 2009. Associations between the 11-bp deletion in the myostatin gene and carcass quality in Angus-sired cattle. Animal Genetics 40, 97-100.

Gong, H., Zhou, H., Hickford, J.G.H., 2011. Diversity of the glycine/tyrosine-rich keratinassociated protein 6 gene (KAP6) family in sheep. Molecular Biology Reports 38, 31-35.

Greenwood, T.A., Kelsoe, J.R., 2003. Promoter and intronic variants affect the transcriptional regulation of the human dopamine transporter gene. Genomics 82, 511-520.

Haidet, A.M., et al., 2008. Long-term enhancement of skeletal muscle mass and strength by single gene administration of myostatin inhibitors. Proceedings of the National Academy of Sciences of the United States of America 105, 4318-4322.

Hickford, J.G., et al., 2010. Polymorphisms in the ovine myostatin gene (MSTN) and their association with growth and carcass traits in New Zealand Romney sheep. Animal Genetics 41, 64-72.

Hill, J.J., Qiu, Y., Hewick, R.M., Wolfman, N.M., 2003. Regulation of Myostatin in vivo by growth and differentiation factor-associated serum protein-1: a novel protein with protease inhibitor and follistatin domains. Molecular Endocrinology 17, 1144-1154.

Jung, H.S., et al., 1998. Local inhibitory action of BMP and their relationships with activators in feather formation: implications for periodic patterning. Developmental Biology 196, 11-23.

Kambadur, R., Sharma, M., Smith, T.P., Bass, J.., 1997. Mutations in myostatin (GDF8) in double-muscled Belgian Blue and Piedmontese cattle. Genome Research 7, 910-916.
Kondas, K., Szlama, G., Trexler, M., Patthy, L., 2008. Both WFIKKN1 and WFIKKN2 have high affinity for growth and differentiation factors 8 and 11. The Journal of Biological Chemistry 283, 23677-23684.

Kulessa, H., Turk, G., Hogan, B.L., 2000. Inhibition of Bmp signaling affects growth and differentiation in the anagen hair follicle. The EMBO Journal 19, 6664-6674.

Lee, Y., Lee, S., 2013. Regulation of GDF-11 and myostatin activity by GASP-1 and GASP-2. Proceedings of the National Academy of Sciences of the United States of America 110, E3713-E3722.

Marcelo, S., Antonio, G., Elen, H., Igor, L., Anselmo, S., 2009. Expression of genes related to myostatin signaling during rat skeletal muscle longitudinal growth. Muscle \& Nerve 40, 992-999.

McDowall, M., Edwards, N.M., Jahoda, C.A.B., Hynd, P.I., 2008. The role of activins and follistatins in skin and hair follicle development and function. Cytokine \& Growth Factor Reviews 19, 415-426.

McPherron, A.C. Lawler, A.M. Lee, S.J. 1999. Regulation of anterior/posterior patterning of the axial skeleton by growth/differentiation factor 11 . Nature Genetics 22, 260-264.

Monestier, O., et al., 2012. Ubiquitous Gasp1 overexpression in mice leads mainly to a hypermuscular phenotype. BMC Genomics 13, 541.

Mosher, D.S., et al., 2007. A mutation in the myostatin gene increases muscle mass and enhances racing performance in heterozygote dogs. PLoS Genetics 3, e79.

Muir, P.D. Cruickshank, G.J., Wallace, G.J., MacLean, K.S., Smith, N.B., 1992. Effects of grazing pressure and competition on growth rate of Merino and Romney lambs. Proceedings of the New Zealand Society of Animal Production 52, 247-250.

Noramly, S., Morgan, B.A., 1998. BMP mediate lateral inhibition at successive stages in feather tract development. Development 125, 3775-3787.

Szlama, G., Kondas, K., Trexler, M., Patthy, L., 2010. WFIKKN1 and WFIKKN2 bind growth factors TGFb1, BMP2 and BMP4 but do not inhibit their signaling activity. The FEBS Journal 277, 5040-5050.

Tamura, K., Dudley, J., Nei, M., Kumar, S., 2007. MEGA4: Molecular Evolutionary Genetics Analysis (MEGA) software version 4.0. Molecular Biology and Evolution 24, 1596-1599.

Trexler, M., Banyai, L., Patthy, L., 2001. A human protein containing multiple types of protease-inhibitory modules. Proceedings of the National Academy of Sciences of the United States of America 98, 3705-3709.

Trexler, M., Bányai, L, Patthy, L, 2002. Distinct expression pattern of two related human proteins containing multiple types of protease-inhibitory modules. Biological Chemistry $383,223-228$.

Zhang, B., Zhao, G., Lan, X., Lei, C., Zhang, C., Chen, H., 2012. Polymorphism in GHRH gene and its association with growth traits in Chinese native cattle. Research in Veterinary Science 92, 243-246.

Zhou, H., Hickford, J.G.H., Fang, Q., 2006. A two-step procedure for extracting genomic DNA from dried blood spots on filter paper for polymerase chain reaction amplification. Analytical Biochemistry 354, 159-161. 